\title{
National Institute of Diabetes and Digestive and Kidney Diseases
}

National Cancer Institute

\section{Source}

National Cancer Institute. National Institute of Diabetes and Digestive and Kidney

Diseases. NCI Thesaurus. Code C19756.

An institute within the National Institutes of Health that conducts and supports much of the clinical research on the diseases of internal medicine and related subspecialty fields, as well as many basic science disciplines. 